\title{
Associated Persons Laboratory Test Results Domain
}

National Cancer Institute

\section{Source}

National Cancer Institute. Associated Persons Laboratory Test Results Domain. NCI

Thesaurus. Code C147172.

A domain utilized for the submission of laboratory test records related to persons associated with the study subject. 